\title{
A checking method for probabilistic seismic-hazard assessment: case studies on three cities
}

\author{
Hing-Ho Tsang $\cdot$ Saman Yaghmaei-Sabegh $\cdot$ P. Anbazhagan • \\ M. Neaz Sheikh
}

Received: 8 July 2010/Accepted: 9 October 2010/Published online: 3 November 2010

(C) The Author(s) 2010. This article is published with open access at Springerlink.com

\begin{abstract}
The conventional Cornell's source-based approach of probabilistic seismichazard assessment (PSHA) has been employed all around the world, whilst many studies often rely on the use of computer packages such as FRISK (McGuire FRISK-a computer program for seismic risk analysis. Open-File Report 78-1007, United States Geological Survey, Department of Interior, Washington 1978) and SEISRISK III (Bender and Perkins SEISRISK III - a computer program for seismic hazard estimation, Bulletin 1772. United States Geological Survey, Department of Interior, Washington 1987). A "black-box" syndrome may be resulted if the user of the software does not have another simple and robust PSHA method that can be used to make comparisons. An alternative method for PSHA, namely direct amplitude-based (DAB) approach, has been developed as a heuristic and efficient method enabling users to undertake their own sanity checks on outputs from computer packages. This paper experiments the application of the DAB approach for three cities in China, Iran, and India, respectively, and compares with documented results computed by the source-based approach. Several insights regarding the procedure of conducting PSHA have also been obtained, which could be useful for future seismichazard studies.
\end{abstract}

Keywords Probabilistic · Seismic-hazard assessment · Hong Kong · Tehran · Bangalore · Ground motion prediction equation

H.-H. Tsang $(\bowtie)$

Department of Civil Engineering, The University of Hong Kong, Pokfulam Road, Hong Kong

e-mail: tsanghh@hku.hk

S. Yaghmaei-Sabegh

Department of Civil Engineering, University of Tabriz, Tabriz, Iran

P. Anbazhagan

Department of Civil Engineering, Indian Institute of Science, Bangalore, India

M. Neaz Sheikh

Department of Civil, Mining and Environmental Engineering, University of Wollongong, Wollongong, Australia 


\section{Introduction}

Seismic-hazard assessment provides an estimate of ground motion at the site of interest, taking into account instrumental and historical earthquake records, information on tectonics, geology, and attenuation characteristics of seismic waves. Seismic-hazard assessment is also used for seismic microzonation study, which is important for decision-making on land use, evaluation of the level of earthquake preparedness, economical consideration of earthquake-resistant design, retrofit strategy, economic loss estimation in an event of future earthquake, and also for the design of ordinary structures where site-specific studies are not warranted.

There are two methodologies of seismic-hazard assessment, namely, deterministic seismic-hazard assessment (DSHA) and probabilistic seismic-hazard assessment (PSHA) (Reiter 1990). In DSHA, ground motion parameters are estimated from a single earthquake event (normally with maximum considered earthquake) or a couple of potential critical earthquake events, without explicitly considering the probability of occurrence within the exposure period considered. However, selection of the representative earthquakes is difficult and the lack of probabilistic information precludes its application in risk analysis and performance-based seismic design.

On the contrary, the effects of all potential earthquake events expected to occur within a specific exposed period are integrated in PSHA with due consideration of the uncertainties and randomness. PSHA is thus able to provide an estimate of ground motion parameters with an annual probability of exceedance (or any other time period), which is a key input for risk analysis and performance-based seismic design (McGuire 2004). PSHA has been conducted all around the world, whilst many studies often rely on the use of computer packages such as FRISK (McGuire 1978) and SEISRISK III (Bender and Perkins 1987), which may require special training to use and lack transparency. A "black-box" syndrome may be resulted if the user of the software does not have another simple and robust PSHA method that can be used to make comparisons.

An innovative alternative method for PSHA, namely direct amplitude-based (DAB) approach (Tsang and Chandler 2006), has been developed as a heuristic and efficient method enabling users to undertake their own sanity checks on outputs from computer packages. In this paper, existing methods for PSHA have been briefly reviewed (Sect. 2). Detailed description of the DAB approach has been given in Sect. 3. This paper experiments the application of the DAB approach for three cities in China, Iran, and India, respectively, and compares with documented results from previous seismic-hazard studies (Sects. 4 and 5). It is not the intention of the authors to override the results computed by other methods (noted that some authors of this paper also involved in those previous studies). This paper only serves as sanity checks on outputs from computer packages. Meanwhile, several insights regarding the procedure of conducting PSHA have also been obtained, which could be useful for future seismic-hazard studies.

\section{Review of existing PSHA methods}

\subsection{Cornell (1968)}

The most commonly employed approach for PSHA is that developed originally by Cornell (1968). This approach incorporates the influence of all potential sources of earthquakes and their corresponding activity rates. The concept of a potential source of earthquakes plays a 
very important role in this methodology. A potential source of earthquakes, which can be in the form of a point, a fault, or a region, is a location where future earthquakes may occur. To describe a potential source of earthquakes, one must decide its form, size, boundary, and the activity rates of earthquakes of different magnitudes. Hence, this method is fundamentally a source-based approach. As this approach is considered difficult to conduct for regions lacking adequate information on the source characteristics, or with a paucity of recorded strong motion data, various alternative procedures have been developed.

\subsection{McGuire (1993)}

McGuire (1993) has proposed a so-called historic method, which is based on historical earthquake events and does not involve characterization of sources. The major assumption of this method is that future seismicity at a particular site can be statistically represented by its seismic history. For each historical earthquake, the probability distribution of ground motion is estimated. By summing up the distribution functions of all historical earthquakes, followed by dividing the whole function by the number of years of the historical catalogue, the annual rates at which different levels of ground motion are exceeded can be obtained. Despite its simplicity in application, the major disadvantage of the historic method is its unreliability at low probability, especially for low-seismicity regions where major events might not exist in the earthquake catalogue.

\subsection{Frankel (1995)}

Frankel (1995) has developed a method for the United States national seismic-hazard mapping program that eliminated the need to characterize seismic sources as well. For regions far from identified active faults, the probabilistic amplitude calculation was based on smoothed historical seismicity. The uncertainties associated with the historical catalogue, such as the location error, could be reduced by smoothing the historical seismicity spatially to different length scales. The selection of the correlation distance $c$ assumed for the Gaussian function in the smoothing process is somehow arbitrary, and a further sensitivity analysis might be required.

\subsection{Kijko and Graham (1999)}

Kijko and Graham (1999) have proposed a "parametric-historic" procedure for PSHA, which does not require the characterization of seismic sources either, whilst retains some features of the conventional source-based method. The advantage of this method is that earthquake catalogues of different completeness conditions could be combined in the hazard assessment, rendering it suitable for region where the seismicity is not well understood. A limitation has however been posed on the choice of ground motion prediction equations (GMPEs) that does not allow non-linear magnitude scaling.

\subsection{Tsang and Chandler (2006)}

Tsang and Chandler (2006) presented a direct amplitude-based (DAB) approach, derived analytically from Cornell's source-based method, yet does not require detailed characterization of seismic sources. Whilst the method possesses the simplicity of the historic 
method, it could be extended to account for characteristic earthquakes and potential large events that have not been observed historically, in order to improve the reliability of hazard calculation at low probability. Hence, it can also be regarded as a "parametric-historic" method. On the other hand, any site-specific and event-specific characteristics that influence ground motions, such as non-linear site effects, rupture mechanism and directivity, can be incorporated in the early stage of the numerical procedure, which is considered beneficial for microzonation study. However, seismicity could not be smoothed spatially by this approach. Detailed description of DAB approach has been given in the following section.

\section{Direct amplitude-based (DAB) approach}

\subsection{Analytical framework}

The source-based approach (Cornell 1968; Reiter 1990; McGuire 2004) can be analytically represented by Eqs. 1 and 2. The effects of all earthquakes of different sizes, occurring at different locations within different earthquake sources and having various probabilities of occurrence, are integrated into a single seismic-hazard curve that shows the probability of ground shaking level $Z$ exceeding $z$ at the site during a specified period of time $T$, as follows:

$$
P[Z>z]=1-e^{-v(z) T} \leq v(z) T
$$

where $v(z)$ is the mean annual rate of exceedance during the time period $T$ and is given by:

$$
v[z]=\sum_{i=1}^{N_{s}} \mu_{i} \int_{M=M_{0}}^{M=M_{u}} \int_{R=0}^{R=\infty} P[Z>z \mid M, R] f_{i}(R \mid M) f_{i}(M) d R d M
$$

where $\mu_{i}$ is the mean annual rate of occurrence of earthquakes between threshold and maximum magnitudes $\left(M_{0}\right.$ and $\left.M_{u}\right)$ being considered in the $i$-th source; $P[Z>z \mid M, R]$ is the probability that the ground shaking level $Z$ of a given earthquake with magnitude $M$ and source-site (or epicentral) distance $R$ will exceed $z ; f_{i}(M)$ is the probability density function $(P D F)$ of magnitude within the $i$-th source; $f_{i}(R \mid M)$ is the $P D F$ of source-site (or epicentral) distance that is conditional on magnitude $M$, describing the spatial distribution between the various locations within the $i$-th source; and $N_{s}$ is the number of sources being considered.

The alternative method, direct amplitude-based (DAB) approach, was developed based on the analytical framework of the source-based approach, using the idea of considering an infinite number of sources, i.e. $N_{s} \rightarrow \infty$ in Eq. 2. In effect, every finite point can be considered as a "source," assuming that there is no repetition of earthquake occurrence at any individual point. The DAB approach can be analytically represented by Eq. 3, and details of the derivation process can be found in Tsang and Chandler (2006).

$$
v[z]=N\left(\Delta_{\min }\right) \int_{\Delta_{\min }}^{\Delta_{\max }} P[Z>z \mid \Delta] f(\Delta) d \Delta
$$

where $\Delta$ is the median ground motion or spectral response amplitude, obtained from GMPEs for each earthquake scenario (discarding the standard deviation); $f(\Delta)$ is the $P D F$ 
of the median amplitude $(\Delta)$, which can be obtained by differentiating the cumulative distribution function $(C D F)$, derived from the amplitude-recurrence relationship. Details of the amplitude-recurrence relationship are given in the following section. $\Delta_{\min }$ and $\Delta_{\max }$ are minimum and maximum median ground motion or spectral response amplitudes, respectively, and $N\left(\Delta_{\min }\right)$ is the mean annual rate of the median amplitude $(\Delta)$ exceeding the minimum value $\left(\Delta_{\min }\right)$. The rationale of maximum median amplitude $\Delta_{\max }$ will be discussed in the following section as well.

The steps involved in this approach are shown in Fig. 1. It can be seen that there is no need to characterize seismic sources, because all events that significantly affect the site are included in the analysis, without considering the spatial distribution of seismicity.

\subsection{Amplitude-recurrence relationship}

In the source-based approach, developing a magnitude-recurrence relationship is the prerequisite, as its derivative is the $P D F$ of magnitude $f_{i}(M)$ in Eq. 2. In the DAB approach, a similar recurrence relationship has been proposed by using the median ground motion or spectral response amplitude $\Delta_{j}$ as the subject parameter. The validity of such recurrence relationship has been shown in an early study by Ishimoto and Iida (1939) using earthquake data recorded in Japan from 1924 to 1937. Similar amplitude-recurrence method has

\section{Step 1: Earthquake Catalog}

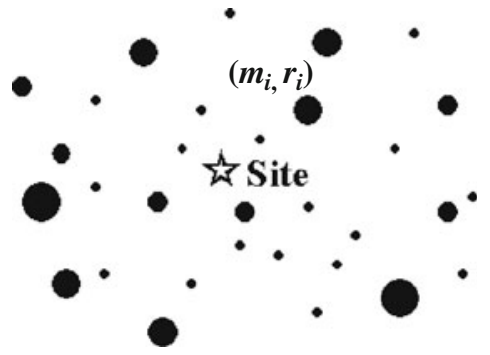

Map of earthquake epicenters with associated magnitudes $m_{i}$ and distances $r_{i}$

\section{Step 3: Amplitude Recurrence}

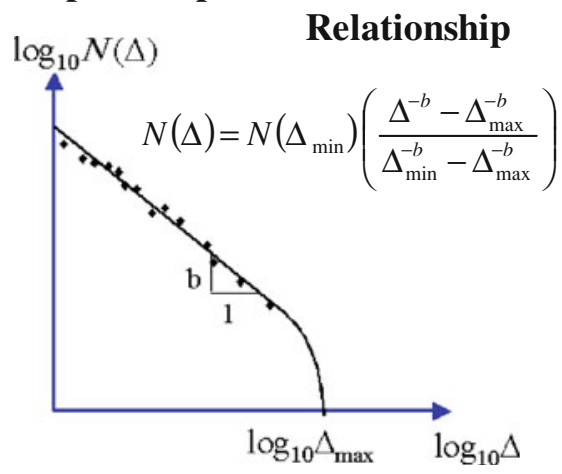

\section{Step 2: Ground Motion} Attenuation Modeling for Each Event

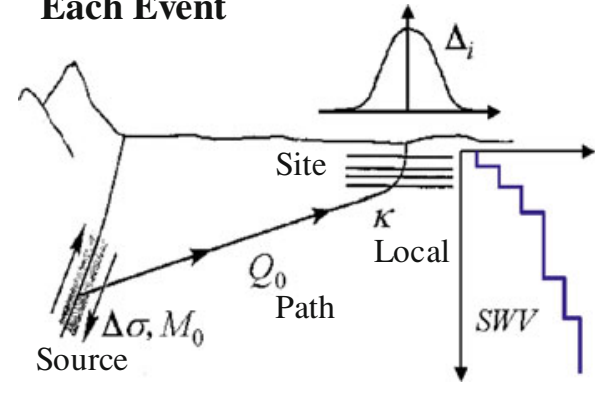

Fig. 1 Steps involved in the direct amplitude-based (DAB) approach of PSHA (Tsang and Chandler 2006)

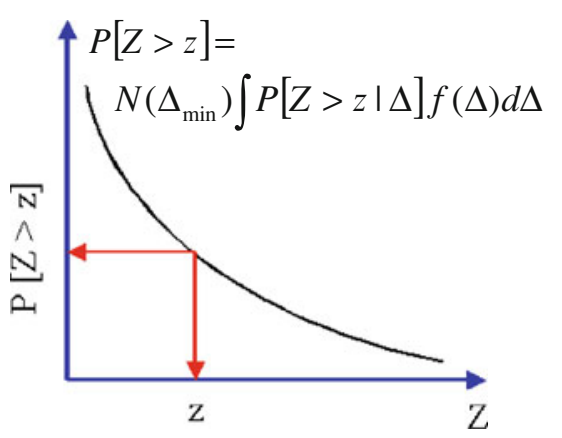


also been adopted by Milne and Davenport (1969) in an earthquake-hazard study for Canada, which was based on counting the annual number of exceedances of a specified acceleration at a site. The use of median peak ground motions (acceleration and velocity) and spectral response amplitudes of a wide range of natural periods (0.01-5.0 s) has further been validated in a recent seismic-hazard study for Hong Kong (Tsang and Lam 2010).

However, it is likely that the earthquake catalogues used are complete for different time periods at different magnitude or intensity levels, which is an important issue that has not been explicitly considered in the historic method. Hence, median ground motion amplitudes of all historical earthquakes in each catalogue (of certain magnitude range and period of time) can be computed (refer Step 2 in Fig. 1), followed by normalizing the amplituderecurrence rates of each catalogue to the same period of time (e.g., 1 year). Then, a single amplitude-recurrence relationship could be obtained by summing up the normalized recurrence rates from all catalogues (refer Step 3 in Fig. 1).

For example, a doubly truncated exponential recurrence relationship for the logarithm of the median amplitude $\left(\log _{10} \Delta\right)$ could be employed, with the consideration of maximum $\left(\Delta_{\max }\right)$ and minimum values $\left(\Delta_{\min }\right)$ (Tsang and Chandler 2006). The maximum value $\left(\Delta_{\text {max }}\right)$ can be used to account for potential large events that have not been observed historically. Determining this maximum median amplitude $\left(\Delta_{\max }\right)$ would be similar to performing deterministic seismic-hazard assessment and is also similar to the definition of the maximum magnitude for each source in the source-based approach (Cornell 1968). Hence, the full range of possible earthquakes that could generate strong ground shaking at the site can be captured. This can then improve the reliability of the historical method at low probability, although certain level of subjectivity would be anticipated in the estimation. The recurrence rate of the characteristic earthquake on a specific fault can also be explicitly assessed based on palaeoseismic observation and crustal strain data (Schwartz and Coppersmith 1984).

Nevertheless, any appropriate form of recurrence relationship can be chosen, depending on the data collected, whilst no restriction has been imposed herein.

If the doubly truncated exponential recurrence relationship is employed for the logarithm of the median ground motion or spectral response amplitude, the number of events leading to the median amplitude $\Delta$ exceeding certain value would be

$$
N(\Delta)=N\left(\Delta_{\min }\right)\left(\frac{\Delta^{-b}-\Delta_{\max }^{-b}}{\Delta_{\min }^{-b}-\Delta_{\max }^{-b}}\right)
$$

from which the $C D F$ of the median ground motion or spectral response amplitude can be expressed as

$$
F(\Delta)=\frac{\Delta_{\min }^{-b}-\Delta^{-b}}{\Delta_{\min }^{-b}-\Delta_{\max }^{-b}}
$$

Further, the $P D F$ can be obtained by differentiating the $C D F$ with respect to $\triangle$.

$$
f(\Delta)=\frac{b \Delta^{-(b+1)}}{\Delta_{\min }^{-b}-\Delta_{\max }^{-b}}
$$

For the $b$-parameter, maximum likelihood estimation has been adopted. The $b$-parameter for each amplitude-recurrence relationship may be obtained from 


$$
b=\frac{\bar{\Delta}}{\bar{\Delta}-\Delta_{\min }}
$$

where $\bar{\Delta}$ is the mean or the expected value of $\Delta$.

\subsection{Analytical (closed-form) solution}

The Cornell's source-based approach, as shown in Eq. 2, involves an integration process, with respect to two main variables, namely magnitude $M$ and distance $R$, which normally requires lengthy computation and is carried out by means of computer programs. However, limitations on the uses of the attenuation relationships and the choices of geographical source types may exist in the available computer programs, which may not be able to cover some complex cases. Ordaz (2004) has provided closed-form solutions to avoid lengthy computations. Owing to the same limitations, closed-form solutions can only be provided for some simple cases that have mainly been used to check the accuracy of the computer programs.

In this section, a generic analytical solution has been derived for the DAB approach. As there is no specification of seismic sources and also the median ground motion amplitudes are computed before performing the integration, the integration process would be free from the aforementioned limitations, which can give the closed-form solution a generic nature. Hence, the DAB approach, along with the analytical solution, can serve as a simplified method for users to undertake their own sanity checks on outputs from other computer packages.

Moreover, for the source-based method to consider non-linear site effects (e.g. Tsai 2000; Cramer 2003), the integration has to be performed with respect to one additional variable, the bedrock ground motion, which would further increase the number of integration steps, and hence computation effort. However, in the proposed DAB approach, any event-specific and site-specific effect, including non-linear site response, can be incorporated at an earlier stage of the numerical procedures. Hence, the generic analytical solution proposed herein can still be applied, without any modification. This forms a significant additional advantage of the $\mathrm{DAB}$ approach, with its generic closed-form solution as shown as follows.

$$
P[Z>z]=\frac{\eta}{z^{b}}\left\{\exp \left(\frac{m^{2}}{2}\right)\left[D(u+m)-\frac{1}{2}\right]-\exp (-m u) D(u)\right\}_{\Delta_{\min }}^{\Delta_{\max }}
$$

where $D(u)$ is the $C D F, u=\frac{1}{\sigma} \log \frac{\Delta}{z}, m=\frac{b \sigma}{\log e}$ and $\eta=\frac{N\left(\Delta_{\min }\right)}{\Delta_{\min }^{-b}-\Delta_{\max }^{-b}}$.

The credibility of the DAB approach has already been demonstrated in Tsang and Chandler (2006), in which peak ground velocity (PGV) has been adopted as the "amplitude".

\section{Case studies on three cities}

The DAB approach has been experimented for three cities in China, Iran, and India, respectively, by comparing the outputs with documented results computed by the sourcebased approach. It is not the intention of the authors to override the results computed by other methods (noted that some authors also involved in those previous studies). This paper only serves as sanity checks on outputs in those documented studies. Meanwhile, several 
insights regarding the procedure of conducting PSHA have also been obtained, which could be useful for future seismic-hazard studies.

\subsection{Hong Kong, China}

Hong Kong is situated in south-east China near the south-eastern margin of the Eurasian Continental Plate in a stable continental intraplate region about $700 \mathrm{~km}$ from the nearest plate boundary, which underlies Taiwan and trends south to the Philippines and north-east to Japan. Although Hong Kong is located in a region of low-to-moderate seismicity, the possibility of a major earthquake in or near the territory cannot be ruled out. The area of Dangan Islands, $30 \mathrm{~km}$ south-east of Hong Kong, was identified by the China Earthquake Administration as a potential source of earthquakes of up to moment magnitude of 7.5 (Chau et al. 2004). However, seismic design has yet to be specifically required in the current building design codes in Hong Kong.

Stochastic simulations of the seismological model, with the consideration of site-specific (rock site) and event-specific characteristics, were performed for each historical event surrounding Hong Kong. The limit of maximum source-site distance of the earthquake database was decided by considering the seismicity pattern of the region surrounding Hong Kong. Seismic activity rates are significantly higher at distances exceeding $600 \mathrm{~km}$ from Hong Kong, where large magnitude earthquake events $(M>7)$ have occurred more frequently. As the ground motion (in terms of PGV) of an event with $M=7.5$ and $R=1,000 \mathrm{~km}$ is comparable to that of an event with $M=6$ and $R=350 \mathrm{~km}$, the limit for the maximum source-site distance of earthquake events collected has been set as $1,500 \mathrm{~km}$ in this study. Also, the minimum magnitudes are taken as $M=3.5$ and $M=6$, for $R<500 \mathrm{~km}$ and $R>500 \mathrm{~km}$, respectively. The regional average crustal conditions have been employed, with details of the input parameters contained in Chandler et al. (2005a, b; $2006 \mathrm{a}, \mathrm{b})$. On the other hand, to capture the range of possible large earthquakes, three independent studies have been employed (Chandler and Lam 2002; OAP/BD 2004; Chau et al. 2004) in defining the maximum median (PGV) amplitude. The results based on the three scenarios have been equally weighted by a logic-tree approach to capture the epistemic uncertainty.

After obtaining the PGV-recurrence relation by Eq. 4, the analytical solution (Eq. 8) has been employed to compute the probabilities of exceeding different levels of PGV and hence form a seismic-hazard curve. The standard deviation $\sigma_{\log (\mathrm{PGV})}($ base 10) employed in this study is 0.3 , which is on a higher side of the typical range of values collected globally by Douglas (2003). Also, this value is consistent with the combined aleatory and epistemic standard deviation derived in Campbell (2003) for the hybrid empirical attenuation relations in eastern North America, which has also employed a seismological modelling approach.

Figure 2 shows the seismic-hazard curves computed separately for the three proposals of maximum median amplitudes. The return period is plotted against PGV, where the former has been computed by taking the reciprocal of the annual probability of exceedance. The results for the return period up to 2,475 years are very similar, and significant deviation can only be observed at rather low probabilities, with about $10 \%$ difference in the hazard predictions at a return period of 10,000 years. In addition, the PSHA results from Pappin et al. (2008), using the source-based approach, have been superimposed onto Fig. 2. The credibility of the DAB approach has been shown by the consistency of the results. The full potential of the proposed approach could be realized by applying it to soil sites for which the site-to-site variability is more significant. 
Fig. 2 Seismic-hazard curves showing the return period (reciprocal of the annual probability of exceedance) against PGV for Hong Kong, China (Tsang and Chandler 2006). The three cited studies made alternative proposals for estimating maximum median PGV. The three solid circles are the PSHA results from Pappin et al. (2008), using the sourcebased approach

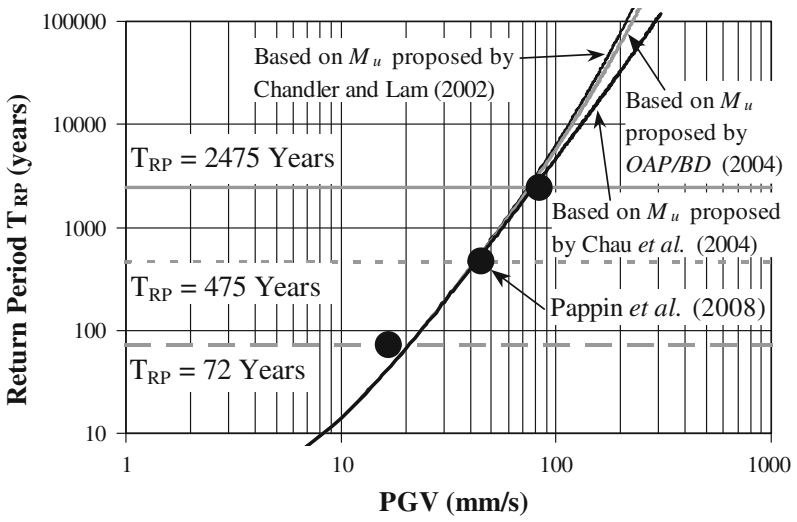

In this study, peak ground acceleration (PGA) has been selected as the "amplitude" for comparison between different cities. Figure 3 shows the doubly truncated PGA-recurrence relationship using Eq. 4. The same three independent studies have been employed for defining the maximum median PGA. The seismic-hazard curves computed separately for the three proposals of maximum median amplitudes have been shown in Fig. 4. The three solid circles are the PSHA results from Pappin et al. (2008), using the source-based approach.

It is seen from Fig. 4 that the hazard values calculated using DAB approach is lower than those calculated by the source-based approach. Similar situation could be seen in Barani et al. (2007) (refer Fig. 8 in that article), in which hazard estimates, in terms of PGA values, computed by the source-based approach are higher than those computed by the spatially smoothed seismicity method (Frankel 1995) that does not require source characterization either. A larger discrepancy can be seen at shorter return period (475 year), as the hazard values at longer return periods are expected to be controlled by the maximum magnitude (in source-based approach) or maximum median (PGA) amplitude (in DAB approach), the more consistent results towards longer return period seem to be reasonable.

\subsection{Tehran, Iran}

The capital of Iran, Tehran city, has been selected for the case study. Iran is situated at the Himalayan-Alpied seismic belt and is one of the high seismic zones in the world. Many

Fig. 3 PGA-recurrence relationship for Hong Kong, China. Three proposals have been made for estimating maximum median PGA

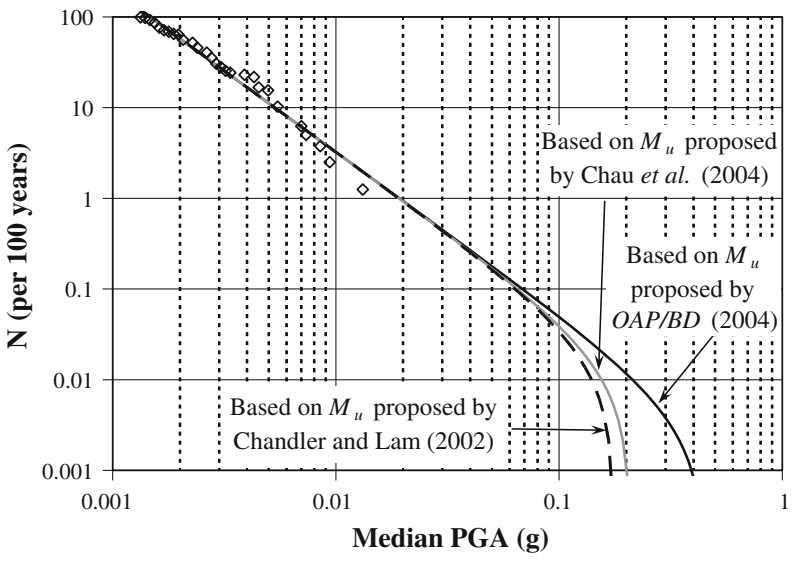


Fig. 4 Seismic-hazard curves showing the return period against PGA for Hong Kong, China. The three solid circles are the PSHA results from Pappin et al. (2008), using the source-based approach

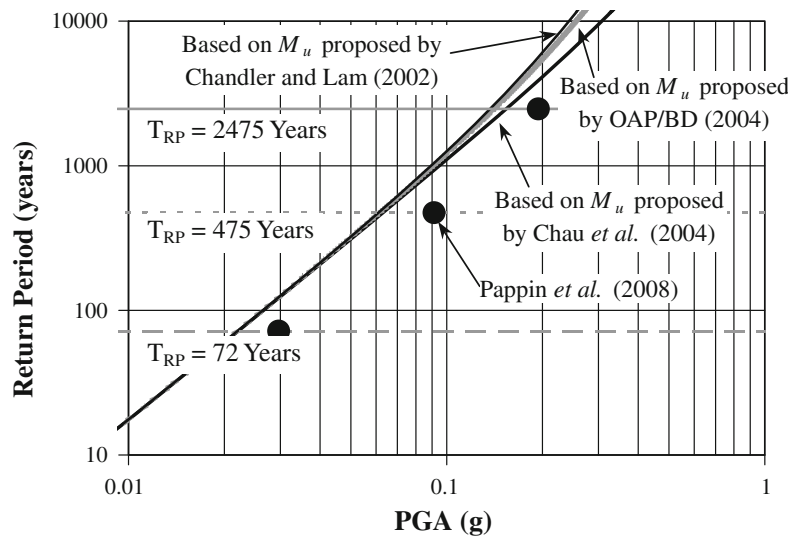

destructive earthquakes occurred in Iran in the past few centuries. Tehran is a densely populated metropolitan city with more than 10 million habitants. It is also the political and economic centre of Iran. Tehran has been severely affected by catastrophic earthquakes for at least six times in the recorded history.

Ghodrati Amiri et al. (2003) have conducted a PSHA for Tehran using the source-based approach, with PGA on rock sites as the ground motion parameter. An earthquake catalogue that contains both historical (before 1900) and instrumental events up to year 2002 has been adopted. Earthquakes occurred within a radius of $200 \mathrm{~km}$ from Tehran were collected and processed. The calculations were performed based on the logic-tree method using three GMPEs for rock sites proposed by Ramazi (1999), Ambraseys and Bommer (1991), and Sarma and Srbulov (1996), with weightings 0.4, 0.35, and 0.25, respectively. As the standard deviation $\sigma_{\log (\mathrm{PGA})}$ (base 10) of all three GMPEs is close to 0.3, a single value of 0.3 has been adopted for all three GMPEs, which is the same as that in the Hong Kong case study.

In order to make direct comparison with the results in Ghodrati Amiri et al. (2003), the same earthquake catalogue and GMPEs have been adopted in this case study using DAB approach. For the maximum median PGA amplitude, two proposals have been adopted. The first one was based on the maximum magnitude of 7.9 adopted in Tavakoli (1996) and supported by Ghodrati Amiri et al. (2003), in which the estimate was $7.8 \pm 0.2$ based on the statistical method proposed by Kijko (2000). Another proposal was based on the earthquake generation capacity of the closest fault-North Tehran fault. Maximum magnitude of 7.0 was estimated based on the fault length, using the empirical formula derived by Nowroozi (1985). The epicentral distance adopted for both proposals is $7.0 \mathrm{~km}$ based on the closest surface distance between the city centre of Tehran and the North Tehran fault. The three GMPEs were also employed for computing maximum median PGA.

Figure 5 shows the doubly truncated PGA-recurrence relationship using Eq. 4. The seismic-hazard curves computed separately for the two proposals of maximum median PGA have been shown in Fig. 6. The hazard values for return period 475 and 975 years calculated by Ghodrati Amiri et al. (2003) have also been superimposed onto Fig. 6. As observed in the Hong Kong case study (Fig. 4), it is seen from Fig. 6 that the hazard values calculated using DAB approach is also lower than those calculated by the source-based approach.

For the large discrepancy at 475 years return period, a possible reason is that in Ghodrati Amiri et al. (2003), uniform seismicity was considered when seismic source 
Fig. 5 PGA-recurrence relationship for Tehran, Iran. Two proposals have been made for estimating maximum median PGA

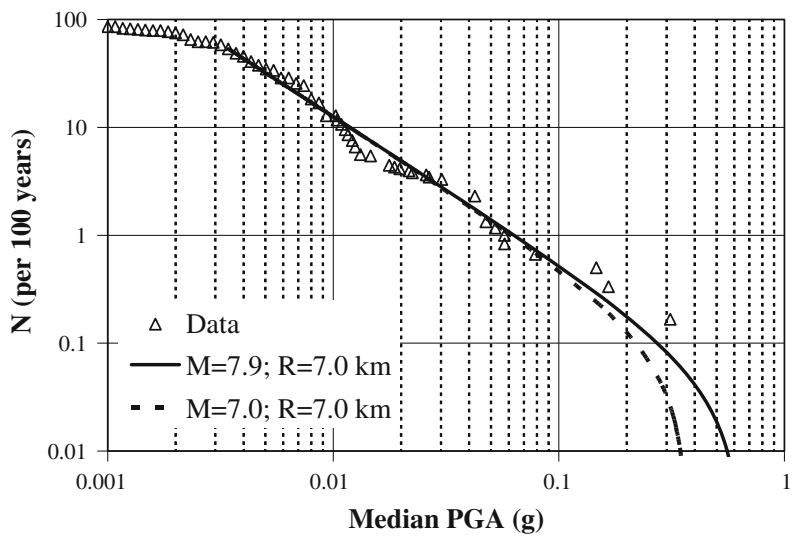

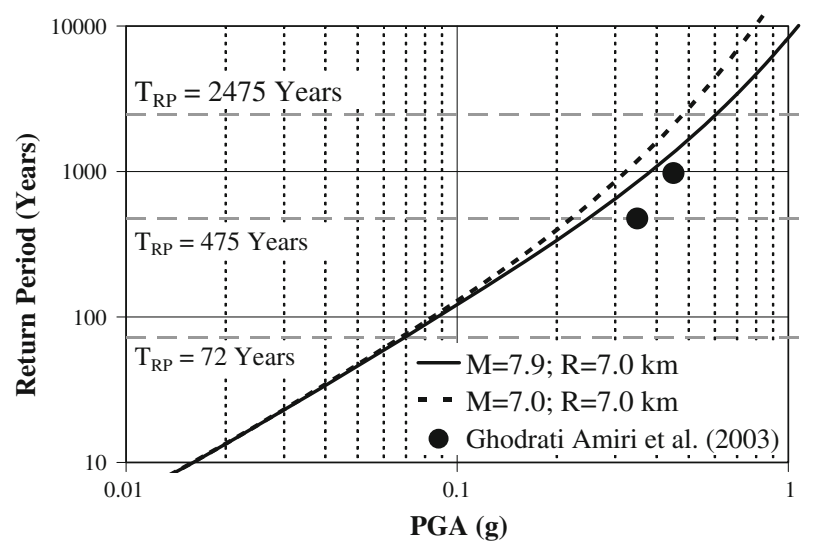

Fig. 6 Seismic-hazard curves showing the return period against PGA for Tehran, Iran. The two solid circles are the PSHA results from Ghodrati Amiri et al. (2003), using the source-based approach

zones were characterized. Hence, unrealistic scenarios might have been considered in the hazard calculation. This includes large magnitude earthquake (say, $7.5<M<8.0$ ) at very short epicentral distance (say, $R<5 \mathrm{~km}$ ) where no fault has been identified. It would overestimate the hazard of the study region. It is also a hidden problem associated with the use of source-based approach if adequate attention has not been paid when characterizing seismic sources. It is recommended that a joint $P D F$ of magnitude and distance, $f(M, R)$, should be adopted in the use of source-based approach.

\subsection{Bangalore, India}

Seismic activity in India is clearly evident from a number of recent earthquakes, which were concentrated along the boundaries of Indo-Australian Plate and Eurasian Plate, as well as within Indo-Australian Plate. In this case study, Bangalore, a city in southern India has been selected. South India has been predominantly considered as a stable continental region; however, numerous earthquakes of magnitude of 6.0 occurred since the eighteenth century and some of which were disastrous. 
Anbazhagan et al. (2009) have conducted a PSHA for Bangalore using the source-based approach, with PGA and spectral acceleration on rock sites as the subject parameters. Uniform hazard response spectrum has also been derived. An earthquake catalogue that contains earthquake events for the period of 1807-2006 has been used. Analyses have been carried out for the region covering a radius of $350 \mathrm{~km}$ with Bangalore as the centre. GMPE for rock site in the Peninsular India developed by Raghukanth (2005) has been used, with a standard deviation $\sigma_{\log (\mathrm{PGA})}$ (base 10) of 0.14 .

In order to make direct comparison with the results presented in Anbazhagan et al. (2009), the same earthquake catalogue and GMPE have been adopted in this case study using DAB approach. For the maximum median PGA amplitude, two proposals have been adopted. The first one was based on the maximum observed magnitude of around 6.0 in the study region, whilst the second one was the "upper bound" value of $6.5(6.0 \pm 0.5)$, which was estimated using the maximum likelihood approach proposed by Kijko and Sellevoll (1989). The source-site distance adopted for both proposals is $16.0 \mathrm{~km}$ based on the closest hypocentral distance between the city centre of Bangalore and the closest fault-MandyaChannapatna-Bangalore lineaments (epicentral distance of $5.2 \mathrm{~km}$; focal depth of $15 \mathrm{~km}$ ).

Figure 7 shows the doubly truncated PGA-recurrence relationship using Eq. 4. The seismic-hazard curves computed separately for the two proposals of maximum median PGA have been shown in Fig. 8. The PSHA result from Anbazhagan et al. (2009), using the source-based approach, has also been superimposed onto Fig. 8 as shown by the solid rectangle.

The apparent discrepancy between the hazard values calculated using source-based method and DAB approach could be attributed to the following reason: After careful investigations into the earthquake catalogue, it is found that there are abnormally a few data in the period 1901-1966 (refer Table 1, Figs. 3, 4 and 6 in Anbazhagan et al. 2009), which is unusual for such a large region in Southern India. Hence, it is likely that the catalogue is incomplete in this period of time. Also, in the period 1997-2006, instrumental records for small magnitude earthquakes are lacking. Such incompleteness of catalogue would lead to an underestimation of the seismicity rate, if appropriate treatment has not been applied in conducting PSHA. It is noted that in this case study using DAB approach, all events in the above-mentioned two periods have been removed and have not been included in the hazard calculation. The completeness criteria adopted in this study are as follows: $M>5$ for periods 1800-1900 (supported by Anbazhagan et al. 2009) plus

Fig. 7 PGA-recurrence relationship for Bangalore, India. Two proposals have been made for estimating maximum median PGA

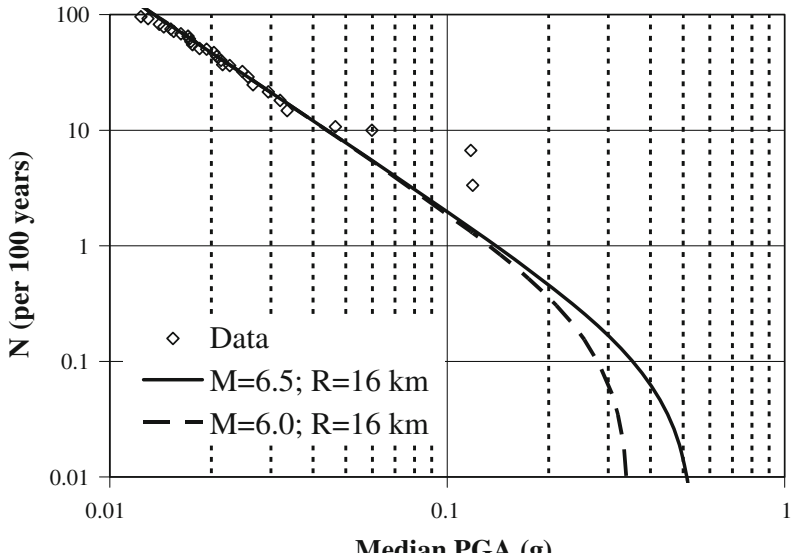

Median PGA (g) 
Fig. 8 Seismic-hazard curves showing the return period against PGA for Bangalore, India. The solid rectangle is the PSHA result from Anbazhagan et al. (2009), using the source-based approach

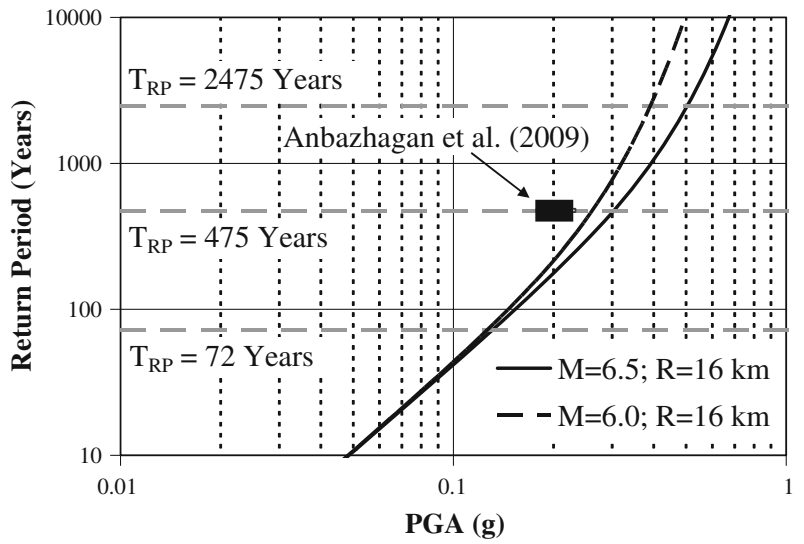

1967-2006 (a total of 140 years) and 5 $>M>3$ for period 1967-1996 (30 years) (supported by Menon et al. 2010).

\section{Comparison of seismic-hazard studies: Hong Kong, Tehran, and Bangalore}

In this section, hazard results obtained for the three cities in Sect. 4 are compared. Figure 9 shows the doubly truncated PGA-recurrence relationships of the three cities considered in this case study. The corresponding seismic-hazard curves have also been shown in Fig. 10. Bangalore curves are based on the maximum median PGA of the earthquake scenario with $M=6.0$ and $R=16.0 \mathrm{~km}$; Hong Kong curves are based on the combined results from the three proposals of maximum median PGA assigned with equal weightings; and the Tehran curves are based on the maximum median PGA of the earthquake scenario with $M=7.9$ and $R=7.0 \mathrm{~km}$.

It is clear that the seismicity and seismic-hazard of Hong Kong are the lowest among the three cities, as both the PGA-recurrence relationship and the hazard curve consistently show lower values of PGA for the whole range of annual activity rate and all return periods.

Interesting results can be observed between Bangalore and Tehran. From the PGArecurrence relationships as shown in Fig. 9, a much higher recurrence rate can be observed

Fig. 9 PGA-recurrence relationships for Hong Kong, Tehran, and Bangalore

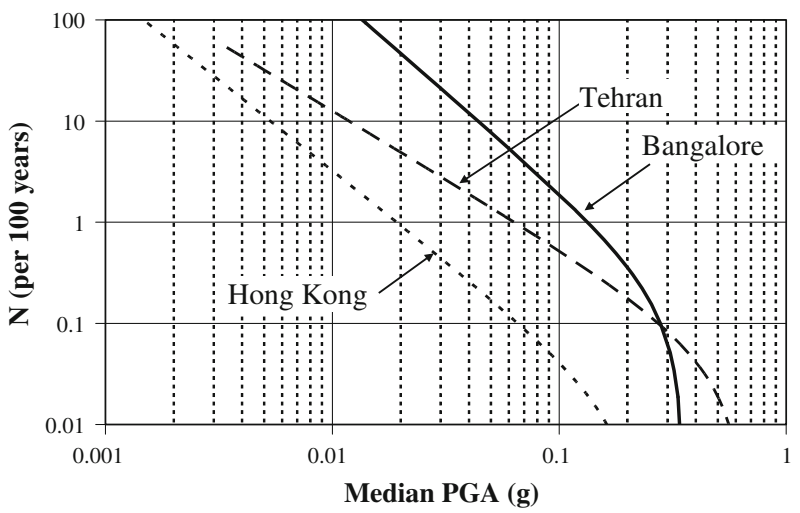


Fig. 10 Seismic-hazard curves showing the return period against PGA for Hong Kong, Tehran, and Bangalore

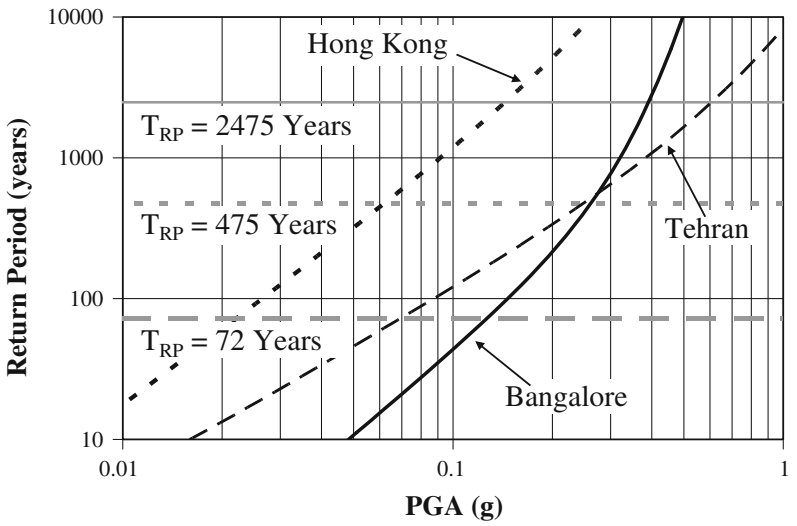

in Bangalore at low shaking levels. The number of events around Tehran that produced a PGA between 0.01 and $0.1 \mathrm{~g}$ is much smaller than that around Bangalore. This may be explained by the extents of the area from which earthquake records have been compiled in the catalogues used for this study. The largest source-site distance of earthquake events in the Tehran catalogue is only $200 \mathrm{~km}$, whilst that of Bangalore and Hong Kong are, respectively, $350 \mathrm{~km}$ and over $500 \mathrm{~km}$. The ratio of area considered in Tehran study to Bangalore study would be 1:3. Although the seismicity of Tehran is expected to be higher, such large ratio would undoubtedly lower the seismic activity rate, especially for low-tomoderate shaking levels, which could be generated by distant earthquakes (with source-site distance greater than $200 \mathrm{~km}$ ). However, a more in-depth study is needed to verify this argument.

On the other hand, the GMPEs adopted for the three cities have been plotted in Fig. 11, and superimposed with Atkinson and Boore (2006) model for hard rock site condition in eastern North America. It is observed that Tehran (weighted) model lies somewhere in between Bangalore and Hong Kong models for $M=5$. However, it is seen that the rate of increase with magnitude of Tehran model is lower than those of the other three GMPEs, of which the rates of increase are fairly similar. In other words, Tehran model predicts a lower PGA for larger magnitude. As the seismicity of Tehran, as well as its maximum median amplitude estimates, relies very much on large magnitude events, the predicted low levels of shaking for large magnitude events reflected by the GMPE of Tehran may result in a lower hazard.

Nevertheless, a crossover point can be seen at PGA of around $0.3 \mathrm{~g}$. The higher activity rate in Tehran at high shaking level is considered reasonable, as earthquakes with large magnitude $(M>7.0)$ can occur at a short distance (say, within $15 \mathrm{~km}$ ), which would in turn result in a larger maximum median amplitude that controls the "tail" (truncated part at high shaking level) of the recurrence relationship. On the contrary, earthquakes occur around Bangalore are considered to be of moderate magnitude $(M<6.0)$ and with distance greater than $15 \mathrm{~km}$.

A closer look at the "tails" (high shaking level) in both Figs. 9 and 10 reveals that the Bangalore and Tehran hazard curves tend to have a wider separation beyond the crossover point, than that in the corresponding recurrence relationships. It can be explained by the much lower standard deviation $\sigma_{\log (\mathrm{PGA})}$ (base 10) of Bangalore GMPE, which has a value of 0.14 , whilst that of Tehran GMPE is equal to 0.3 . If the same value of 0.3 was adopted as the standard deviation of Bangalore GMPE, its hazard predictions, in terms of PGA values, would be increased by around $40 \%$ at return period of 72 years, $60 \%$ at 475 years 
Fig. 11 Ground motion prediction equations (GMPEs) adopted for the three cities, superimposed with Atkinson and Boore (2006) model for hard rock site condition in eastern North America
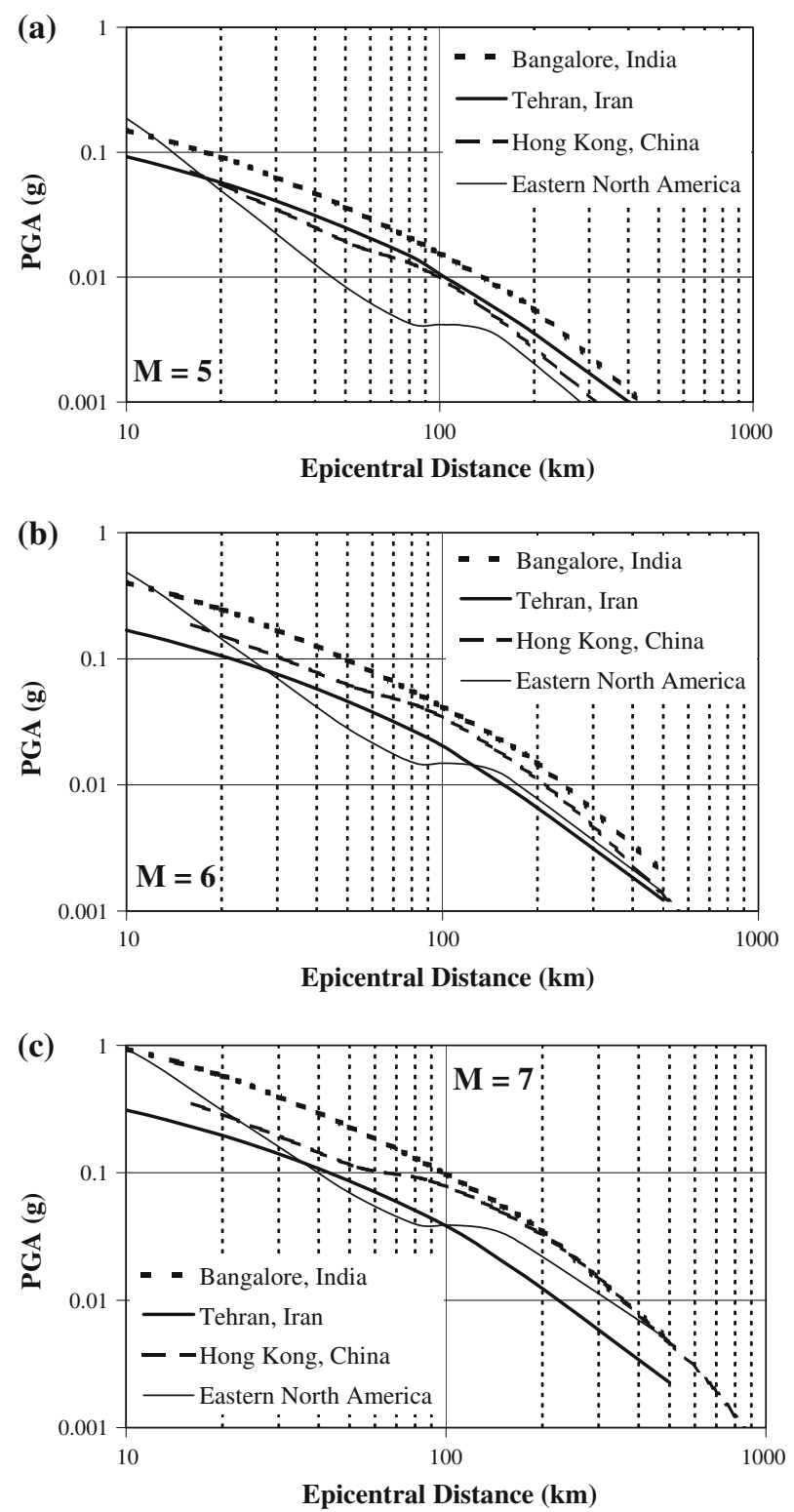

and $80 \%$ at 2,475 years. Hence, the significant influence of the standard deviation on hazard calculation is evidenced, especially at long return period.

\section{Summary and conclusions}

1. A number of methods for probabilistic seismic-hazard assessment (PSHA) have been briefly reviewed. A simpler and more direct method, namely direct amplitude-based 
(DAB) approach, for conducting PSHA has been introduced, which is proposed to serve as a useful tool enabling users to undertake their own sanity checks on outputs from computer packages

2. The advantages of the proposed approach include the following: (1) it does not require detailed characterization of seismic sources; (2) whilst the method possesses the simplicity of the historic method, it is extended to account for characteristic earthquakes and large events that have not been observed historically, in order to improve the reliability of hazard calculation at low probability; (3) any site-specific and event-specific characteristics that influence ground motions can be incorporated in the early stage of the numerical procedure; (4) it does not require lengthy integration process as a generic analytical (closed-form) solution has been derived.

3. Applications of the new method have been conducted for three cities, namely, Hong Kong, China; Tehran, Iran; and Bangalore, India. The results computed by the new method have been compared with previous results computed by source-based approach. The robustness of the proposed checking method has been demonstrated. Several insights regarding the procedure of conducting PSHA have also been obtained as follows.

4. From the Tehran case study, it is suspected that the assumption of uniform seismicity when characterizing seismic sources in the use of the source-based approach may lead to an overestimation of the hazard. This is because some unrealistic scenarios, for instance, large magnitude earthquake (say, $7.5<M<8.0$ ) at very short epicentral distance (say, $R<5 \mathrm{~km}$ ) where no fault has been identified, might have been considered in the hazard calculation. A joint $P D F$ of magnitude and distance, $f(M, R)$, should be employed in the calculation.

5. The completeness of the earthquake catalogue has to be carefully examined. If the catalogue is incomplete in certain period of time and an appropriate treatment has not been applied when conducting PSHA, the seismicity would be underestimated. This might be a reason for the discrepancy in the hazard calculation for Bangalore.

6. The extents of the area from which earthquake records have been compiled in the catalogues may significantly affect the accuracy of the hazard results. Although the seismicity of Tehran is expected to be higher than that of Bangalore, the much smaller area considered in the compilation of the catalogue would undoubtedly lower the seismic activity rate, especially for low-to-moderate shaking levels, which could be generated by distant earthquakes.

7. Reliable prediction of ground motion or spectral response by the ground motion prediction equations (GMPEs) is essential for a credible PSHA. The standard deviation $\sigma_{\log (\mathrm{PGA})}$ of the GMPE would also significantly influence the hazard results, especially at long return period. The lowest rate of increase in the hazard level of Bangalore, compared to those of Hong Kong and Tehran, might be explained by the much lower standard deviation of Bangalore GMPE.

Open Access This article is distributed under the terms of the Creative Commons Attribution Noncommercial License which permits any noncommercial use, distribution, and reproduction in any medium, provided the original author(s) and source are credited.

\section{References}

Ambraseys NN, Bommer JJ (1991) The attenuation of ground accelerations in Europe. Earthquake Eng Struct Dyn 20(12):1179-1202 
Anbazhagan P, Vinod JS, Sitharam TG (2009) Probabilistic seismic hazard analysis for Bangalore. Nat Hazards 48:145-166

Atkinson GM, Boore DM (2006) Earthquake ground-motion prediction equations for eastern North America. Bull Seismol Soc Am 96(6):2181-2205

Barani S, Spallarossa D, Bazzurro P, Eva C (2007) Sensitivity analysis of seismic hazard for western Liguria (north western Italy): a first attempt towards the understanding and quantification of hazard uncertainty. Tectonophysics 435:13-35

Bender B, Perkins DM (1987) SEISRISK III-a computer program for seismic hazard estimation, Bulletin 1772. United States Geological Survey, Department of Interior, USA

Campbell KW (2003) Prediction of strong ground motion using the hybrid empirical method and its use in the development of ground-motion (attenuation) relations in eastern north America. Bull Seismol Soc Am 93:1012-1033

Chandler AM, Lam NTK (2002) Scenario predictions for potential near-field and far-field earthquakes affecting Hong Kong. Soil Dyn Earthq Eng 22:29-46

Chandler AM, Lam NTK, Tsang HH (2005a) Shear wave velocity modelling in crustal rock for seismic hazard analysis. Soil Dyn Earthq Eng 25(2):167-185

Chandler AM, Lam NTK, Tsang HH, Sheikh MN (2005b) Estimation of near-surface attenuation in bedrock for analysis of intraplate seismic hazard. J Seismlog Earthq Eng 7(3):159-173

Chandler AM, Lam NTK, Tsang HH (2006a) Regional and local factors in attenuation modelling: Hong Kong case study. J Asian Earth Sci 27(6):892-906

Chandler AM, Lam NTK, Tsang HH (2006b) Near-surface attenuation modelling based on rock shear-wave velocity profile. Soil Dyn Earthq Eng 26:1004-1014

Chau KT, Lai KW, Wong YL, Wong RHC, Wang LX, Chan YW, Wong WT, Guo YSH, Zhu W (2004) Three-dimensional surface cracking and faulting in Dangan islands area, South of Hong Kong. In: Proceedings of third international conference on continental earthquakes, Beijing, China, 12-14 July 2004

Cornell CA (1968) Engineering seismic risk analysis. Bull Seismol Soc Am 58:1583-1606

Cramer CH (2003) Site-specific seismic-hazard analysis that is completely probabilistic. Bull Seismol Soc Am 93:1841-1846

Douglas J (2003) Earthquake ground motion estimation using strong-motion records: a review of equations for the estimation of peak ground acceleration and response spectral ordinates. Earth Sci Rev 61:43-104

Frankel A (1995) Mapping seismic hazard in the central and eastern United States. Seismol Res Lett 66:8-21

Ghodrati Amiri G, Motamed R, Rabet Es-Haghi H (2003) Seismic hazard assessment of metropolitan Tehran, Iran. J Earthq Eng 7:347-372

Ishimoto M, Iida K (1939) Observations sur les séismes enregistrés par le microsismographe construit dernièrement. Bull Earthquake Res Inst XVII, Tokyo Imperial University, pp 443-478

Kijko A (2000) Statistical estimation of maximum regional earthquake magnitude $m_{\max }$. Workshop of seismicity modeling in seismic hazard mapping, Poljce, Slovenia, 22-24 May 2000

Kijko A, Graham G (1999) "Parametric-historic" procedure for probabilistic seismic hazard analysis—Part II: assessment of seismic hazard at specified site. Pure Appl Geophys 154:1-22

Kijko A, Sellevoll MA (1989) Estimation of earthquake hazard parameters from incomplete data files. Part I: utilization of extreme and complete catalogs with different threshold magnitudes. Bull Seismol Soc Am 79:645-654

McGuire RK (1978) FRISK - a computer program for seismic risk analysis. Open-File Report 78-1007. United States Geological Survey, Department of Interior, USA

McGuire RK (1993) Computations of seismic hazard. Ann Geofis 36:181-200

McGuire RK (2004) Seismic hazard and risk analysis. Earthquake Engineering Research Institute (EERI), Oakland

Menon A, Ornthammarath T, Corigliana M, Lai CG (2010) Probabilistic seismic hazard macrozonation of Tamil Nadu in Southern India. Bull Seismol Soc Am 100(3):1320-1341

Milne WG, Davenport AG (1969) Distribution of earthquake risk in Canada. Bull Seismol Soc Am 59:729-754

Nowroozi A (1985) Empirical relations between magnitude and fault parameters for earthquakes in Iran. Bull Seismol Soc Am 75(5):1327-1338

OAP/BD (2004) The seismic effects on buildings in Hong Kong. Buildings Department, Government of the HKSAR, Consultancy Agreement No. CAO K49, conducted by Ove Arup and Partners (HK) Ltd

Ordaz M (2004) Some integrals useful for probabilistic seismic hazard analysis. Bull Seismol Soc Am $94: 1510-1516$ 
Pappin JW, Koo RCH, Free MW, Tsang HH (2008) Seismic hazard of Hong Kong. Electron J Struct Eng $8: 42-56$

Raghukanth STG (2005) Engineering models for earthquake sources. Dissertation, Indian Institute of Science, Bangalore, India

Ramazi HR (1999) Attenuation laws of Iranian earthquakes. In: Proceedings of third international conference on seismology and earthquake engineering, Tehran, Iran, pp 337-344

Reiter L (1990) Earthquake hazard analysis: issues and insights. Columbia University Press, New York

Sarma SK, Srbulov M (1996) A simplified method for prediction of kinematic soil-foundation interaction effects on peak horizontal acceleration of a rigid foundation. Earthq Eng Struct Dyn 25(8):815-836

Schwartz DP, Coppersmith KJ (1984) Fault behavior and characteristic earthquakes: examples from the Wasatch and San Andreas faults. J Geophys Res 89:5681-5698

Tavakoli B (1996) Major seismotectonic provinces of Iran. International Institute of Earthquake Engineering and Seismology, Tehran, Iran (in Persian)

Tsai CCP (2000) Probabilistic seismic hazard analysis considering nonlinear site effect. Bull Seismol Soc Am 90:66-72

Tsang HH, Chandler AM (2006) Site-specific probabilistic seismic-hazard assessment: direct amplitudebased approach. Bull Seismol Soc Am 96:392-403

Tsang HH, Lam NTK (2010) Seismic hazard assessment in regions of low-to-moderate seismicity. LAP Lambert Academic Publishing, Koln, Germany 